\title{
Atributos físico-hidricos e produtividade de trigo em um Latossolo sob estados de compactação
}

\author{
Physical-hydraulic attributes and wheat yield in an oxisol under compaction states
}

\author{
Andreia Kusumota Bonini ${ }^{\mathrm{I}}$ Deonir Secco ${ }^{\mathrm{II}, \mathrm{I}}$ Reginaldo Ferreira Santos ${ }^{\mathrm{II}}$ Dalvan José Reinert ${ }^{\mathrm{III}}$ \\ José Miguel Reichert ${ }^{\mathrm{II}}$
}

\section{RESUMO}

Este trabalho teve por objetivo avaliar o efeito de estados de compactação sobre os atributos físico-hídricos do solo e na produtividade da cultura do trigo (Triticum spp) em um Latossolo Vermelho Distroférrico sob sistema plantio direto. $O$ cultivo do trigo foi em faixas $(10 \times 50 \mathrm{~m})$ nas quais foram implantados quatro tratamentos correspondentes aos estados de compactação: $S C$-unidade experimental sem passagem do rolo compactador, $1 P$-uma passada com o rolo compactador, $3 P$-três passadas e 5P-cinco passadas. Para avaliar a densidade (Ds), o volume de macroporos (Map) e de microporos (Mip) $e$ a porosidade (Pt) do solo foram coletadas amostras indeformadas nas profundidades de 0,0-0,1, 0,1-0,2 e 0,2$0,3 \mathrm{~m}$, em cinco pontos aleatórios em cada tratamento. Outra amostra foi coletada na camada de 0,0-0,3m, para realizar o ensaio de Proctor normal e determinar a densidade máxima do solo (Dsmax). A taxa infiltração de água no solo foi avaliada por meio de anéis concêntricos, a densidade do solo relativa (Dsrel) foi calculada através da relacão entre a Ds e a Dsmax. Para determinar a produtividade do trigo delimitou-se uma área útil de $4,08 \mathrm{~m}^{2}$, com cinco subamostras em cada tratamento. Com o aumento das passadas do rolo compactador, ocorreu redução da map e incremento da Ds. A produtividade do trigo e a taxa de infiltração de água no solo foram reduzidas, respectivamente, em $23 \%$ e $97 \%$ quando se comparou o tratamento SC em relação ao 5P. A Dsrel ótima para a produtividade do trigo no Latossolo estudado foi de 0,83.

Palavras-chave: compactação do solo, estrutura do solo, plantio direto.

\section{ABSTRACT}

This study aimed to evaluate the effect of additional compaction on the physical-hydraulic attributes and wheat yield (Triticum spp) in an Oxisol under no-tillage. The cultivation was made in strips $(10 \times 50 \mathrm{~m})$ which were implanted in four treatments or states of compaction: SC - without additional compaction with a roller compactor $1 P$ - one roller pass, $3 P$ - tree rolear passes and $5 P$ - five roler passes. To evaluate soil bulk density (Bd), macroporosity (Map), microporosity (Mip) and total porosity (Tp), undisturbed soil samples were collected from three layers: 0,0-0,1, 0,1-0, 2 and 0.2-0.3m, at five random locations in each treatment. Soil samples were also collected in the layer 0.0 to $0.3 \mathrm{~m}$ to perform the Proctor test and determine the maximum soil bulk density (Bdmax). The water infiltration rate in the soil was evaluated by means of concentric rings. The relative soil bulk density (Bdrel) was calculated using the relationship between the Bd and Bdmax. To determine the wheat yield a useful area of $4.08 \mathrm{~m}^{2}$ was delimited and harvested, with five replicates in each treatment. By increasing the number of roller compactor passes, there was a reduction in Map and increase in $B d$. The wheat yield and the infiltration rate decreased, respectively, by $23 \%$ and $97 \%$ when comparing SC treatment on 5 P. The optimal Bdrel wheat yield in this Oxisol was of 0.83 .

Key words: soil compaction, soil structure, no-tillage.

\section{INTRODUÇÃO}

A compactação dos solos agrícolas em áreas manejadas sob sistema plantio direto é um fator limitante para o aumento do rendimento de grãos e para a sustentabilidade deste sistema, especialmente em solos argilosos, que são mais propensos à compactação (SILVA, 2003). O acentuado tráfego de máquinas e equipamentos sobre o solo, em condições

'Curso de Ciências Biológicas, Universidade Estadual do Oeste do Paraná(UNIOESTE), Campus Cascavel, Cascavel, PR, Brasil.

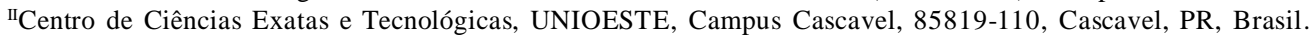

E-mail:deonir.secco@unioeste.br. Autor para corespondência.

II'Departamento de Solos, Centro de Ciências Rurais (CCR), Universidade Federal de Santa Maria (UFSM), Santa Maria, RS, Brasil. 
inadequadas de umidade aliado ao alto peso por eixo, provoca compactação. No entanto, o maior ou menor incremento da compactação depende do estado inicial de compactação, da textura e da umidade que se encontra o solo no momento das atividades agrícolas (SECCO et al., 2009).

Quando o volume de macroporos é inferior a $10 \%$ e a densidade do solo está entre 1,25 e $1,3 \mathrm{Mg} \mathrm{m}^{-3}$, em solo com textura argilosa, há indícios que pode ocorrer restrições ao crescimento radicular e afetar a produtividade das culturas (REICHERT et al., 2009). A determinação de valores críticos para os atributos físicos do solo tem inúmeros obstáculos, pois não são determinantes diretos do crescimento das plantas, diferentemente dos fatores abióticos como ar, água e temperatura, que influenciam diretamente as culturas.

Apesar da densidade do solo e resistência à penetração serem os atributos físicos mais utilizados para monitorar a estrutura do solo, pode-se utilizar também a densidade do solo relativa que tem sido utilizada para caracterizar o estado de compactação do solo, que representa a relação entre as densidades global e máxima do solo (KLEIN, 2006). BEUTLER et al. (2005) estudando estados de compactação na cultura da soja (Glycine max), verificaram que ao passar seis vezes com um trator sobre o solo a densidade obtida foi de $1,80 \mathrm{Mg} \mathrm{m}^{-3} \mathrm{e}$ a densidade do solo relativa ótima de 0,80 em um Latossolo argiloso. Já SUZUKI et al. (2007) afirmam que o valor da densidade do solo relativa ótima para a cultura da soja é de 0,86 .

A taxa de infiltração de água no solo é de extrema importância, pois contribui para a redução do escoamento superficial evitando a ocorrência da erosão do solo. MILANI (2005) estudou a variabilidade espacial da infiltração de água na mesma área objeto deste estudo e encontrou valores de infiltração que variaram de 48 a $396 \mathrm{~mm} \mathrm{~h}^{-1}$. A infiltração de água em Latossolos, em condições normais é geralmente elevada, entretanto esta infiltração pode ser reduzida quando ocorrem camadas compactadas, devido à redução da macroporosidade.

Quanto aos efeitos dos estados de compactação no rendimento de grãos de culturas, SECCO et al. (2009), ao trabalhar com Latossolo Vermelho Distrófico e Latossolo Vermelho Distroférrico, verificaram decréscimo da produtividade do trigo, respectivamente de 18 e $34 \%$, quando a densidade do solo foi maior que $1,50 \mathrm{Mg} \mathrm{m}^{-3}$. O objetivo deste trabalho foi avaliar o efeito de estados de compactação sobre os atributos físico-hídricos do solo e na produtividade da cultura do trigo em um Latossolo argiloso sob sistema plantio direto.

\section{MATERIAL E MÉTODOS}

O experimento foi conduzido durante a safra de trigo no ano de 2006, no Núcleo Experimental de Engenharia Agrícola da UNIOESTE em Cascavel-PR. A área apresenta altitude média de $760 \mathrm{~m}$ e declividade menor que $0,03 \mathrm{~m} \mathrm{~m}^{-1}$. Segundo Köppen o clima da região é temperado mesotérmico e super-úmido (cfa), com precipitação anual em torno de $1920 \mathrm{~mm}$, bem distribuídas na época de verão, com temperatura do ar com média anual em torno de $21^{\circ} \mathrm{C}$. O solo da região é classificado como Latossolo Vermelho Distroférrico típico, textura argilosa a muito argilosa $\left(600 \mathrm{~g} \mathrm{~kg}^{-1} \mathrm{de}\right.$ argila; $320 \mathrm{~g} \mathrm{~kg}^{-1}$ de silte e $80 \mathrm{~g} \mathrm{~kg}^{-1}$ de areia), substrato basalto e relevo suave-ondulado (EMBRAPA, 2006). A área destinada à implantação do estudo foi cultivada por oito anos sob sistema plantio direto, predominantemente com as culturas de aveia (Avena sativa) ou trigo (Triticum spp) no inverno e de milho (Zea mays) ou soja (Glycine max) no verão.

O experimento consistiu na implantação dos tratamentos com estados de compactação, utilizando para isso um rolo compactador, com superfície lisa, dispositivo vibratório ligado, com velocidade de deslocamento em aproximadamente $2,5 \mathrm{~km} \mathrm{~h}^{-1}$ e pressão média de $120 \mathrm{kPa}$. Foi utilizado o cultivo em faixas $(10 \mathrm{x}$ $50 \mathrm{~m}$ ), nas quais foram implantados quatro tratamentos ou estados de compactação: SC-unidade experimental sem passagem do rolo compactador, $1 \mathrm{P}$-uma passada com o rolo compactador, 3P-três passadas e 5P-cinco passadas. No momento da implantação do experimento, essas áreas apresentaram os seguintes conteúdos de água no solo na camada de $0-0,2 \mathrm{~m}$ : SC- $0,37 \mathrm{~kg} \mathrm{~kg}^{-1}, 1 \mathrm{P}-$ $0,37 \mathrm{~kg} \mathrm{~kg}^{-1}, 3 \mathrm{P}-0,36 \mathrm{~kg} \mathrm{~kg}^{-1}$ e $5 \mathrm{P}-0,38 \mathrm{~kg} \mathrm{~kg}^{-1}$. Cada faixa com ou sem compactação foi considerada como sendo o tratamento, tendo em vista que as coletas que configuraram as repetições ocorriam dentro das mesmas.

Antes e após a implantação do experimento (abril de 2006), a caracterização de atributos físicos do solo foi realizada com base na metodologia da EMBRAPA (1997), na qual foram analisados: a densidade do solo (Ds) e de partículas (Dp), macroporosidade (Map), microporosidade (Mip) e a porosidade total $(\mathrm{Pt})$. Em cada tratamento foram coletadas amostras de solo em cinco pontos aleatórios, com duas repetições em cada profundidade. A Ds foi avaliada através da coleta de amostras de solo indeformada pelo método do anel volumétrico (diâmetro $=0,05 \mathrm{~m}$ e altura $=0,054 \mathrm{~m}$ ) nas profundidades de $0,0-0,1,0,1-0,2,0,2-0,3 \mathrm{~m}$. O espaço poroso do solo (Pt, Map e Mip) foi determinado nas mesmas profundidades, para avaliar o efeito dos estados de compactação na estrutura do solo. 
Amostras de solo deformadas foram coletadas na profundidade de $0,0-0,30 \mathrm{~m}$ no início do estudo, secadas ao ar e então submetidas à análise de densidade de partículas pelo método do balão volumétrico. Com essa amostra, também se avaliou a densidade máxima do solo (Dsmax), que foi determinada pelo ensaio de Proctor normal conforme preconizado pela NBR 7182 (ABNT, 1986). O valor de Dsmax obtido foi de $1,39 \mathrm{Mg} \mathrm{m}^{-3}$ com umidade de $0,30 \mathrm{~kg} \mathrm{~kg}^{-1}$, valor este utilizado nos procedimentos de cálculos da Dsrel. A Dsrel foi obtida pela expressão: Dsrel = Ds / Dsmax, onde: Dsrel $=$ densidade do solo relativa; $\quad$ Ds $=$ densidade do solo $\left(\mathrm{Mg} \mathrm{m}^{-3}\right)$ e, Dsmax = densidade máxima $\left(\mathrm{Mg} \mathrm{m}^{-3}\right)$.

A taxa de infiltração de água no solo foi determinada após implantação dos tratamentos, com o uso de infiltrômetro de anel duplo, com cinco repetições em cada unidade experimental conforme metodologia preconizada por BERNARDO (1995).

Para a implantação da cultura do trigo, utilizou-se uma semeadora-adubadora de arrasto, com mecanismo sulcador tipo duplo disco defasado para deposição de adubo e sementes. A implantação e manejo seguiram as recomendações técnicas da cultura (REUNIÃ̃O DA COMISSÃO CENTRO-SUL BRASILEIRADE PESQUISADETRIGOETRITICALE, 2005). Para obtenção dos dados de produtividade do trigo, em cada unidade experimental foram delimitadas cinco subamostras com oito linhas de $3 \mathrm{~m}$ de comprimento perfazendo uma área útil de $4,08 \mathrm{~m}^{2}$, sendo que cada subamostra foi considerada uma repetição. Os resultados foram expressos em $\mathrm{Mg} \mathrm{ha}^{-1} \mathrm{e}$ a umidade corrigida para $0,13 \mathrm{~kg} \mathrm{~kg}^{-1}$.

A análise estatística foi realizada considerando-se um delineamento experimental inteiramente casualizado, com quatro tratamentos, definidos pelo número de passadas do rolo compactador, com cinco repetições e constou de comparações entre média de variáveis de solo e da cultura estudados, por meio do teste t de Student a 5\% de significância (BANZATTO \& KRONKA, 1995). Também foi realizada a análise de regressão para a variável Dsrel em relação aos estados de compactação e entre a produtividade e a Dsrel, sendo o grau da equação definido pelo teste $\mathrm{F}$ aplicado a análise de regressão(PIMENTEL GOMES \& GARCIA, 2002). Para verificar as pressuposições da análise de variância, foram realizados os testes de Levene (homogeneidade das variâncias) e Ryan-Joiner e Kolmogorov-Smirnov (normalidade) no software Minitab 5.0.

\section{RESULTADOS E DISCUSSÃO}

O tráfego do rolo compactador resultou em significativas alterações $(\mathrm{P}<0,05)$ na Ds, Map, Mip e Pt
(Tabela 1). A Ds do SC foi inferior e diferiu dos tratamentos 1P, 3P e 5P em todas as profundidades avaliadas, indicando mudanças na estrutura do solo através da pressão aplicada no solo pelo rolo compactador (Tabela 1). Os maiores valores de Ds no sistema plantio direto ocorrem até aproximadamente $0,15 \mathrm{~m}$ de profundidade, devido ao acumulo das tensões impostas pelas máquinas e implementos agrícolas. BEUTLER \& CENTURION (2004), em experimento semelhante a este, com 0, 1, 2, 4 e 6 passadas de trator, num Latossolo Vermelho distrófico, afirmam que ocorreu a formação de uma camada mais compactada na profundidade de 0,07-0,10m e segundo REICHERT et al. (2009), esses valores de Ds encontrados nas camadas de $0,0-0,1$ e $0,1-0,2 \mathrm{~m}$ nos tratamentos $1 \mathrm{P}, 3 \mathrm{Pe}$ $5 \mathrm{P}$, podem ser considerados restritivos ao desenvolvimento da cultura.

Após aplicação dos tratamentos 1P, 3P e 5P apresentaram valores de Dsrel superiores que diferiram do SC em todas as profundidades analisadas, indicando que a Dsrel aumentou em função do estado de compactação do solo. As tensões ocasionadas pelo tráfego de máquinas e implementos agrícolas se intensificam nesta camada, visto que no sistema plantio direto o revolvimento ocorre apenas na camada mais superficial na linha da semeadura. BEUTLER et al. (2005) e SUZUKI et al. (2007) encontraram, respectivamente, valores de Dsrel de 0,80 e 0,86 para o rendimento ótimo da soja e KLEIN (2006) afirma que valores acima de 0,88 são limitantes a produção de grãos.

A Map teve redução nos tratamentos trafegados (1P, 3P e 5P), quando comparado aos valores do tratamento não trafegado (SC), em todas as profundidades analisadas (Tabela 1). Verifica-se também que, na profundidade de $0,0-0,1 \mathrm{~m}$, o volume de Map foi reduzido em torno de 64,73 e $73 \%$, respectivamente, nos tratamentos 1P, 3P e 5P em relação ao SC. Para a profundidade de 0,1-0,2 e 0,2-0,3m observa-se comportamento semelhante, pois o volume de Map sofreu redução maior ou igual a 50\%. Essa diferença no volume de Map se deve aos diferentes valores de Ds que foi encontrado nos estados de compactação. Quando o volume de Map é inferior a $0,1 \mathrm{dm} 3 \mathrm{dm}-3 \mathrm{e}$ $\mathrm{Ds} \geq 1,36 \mathrm{Mg} \mathrm{m}-3$, podem ocorrer limitações no crescimento radicular e, como conseqüência, pode afetar a produtividade das culturas, segundo REICHERT et al. (2009).

Além disso, observou-se que os menores valores de Map encontram-se na profundidade de 0,0$0,2 \mathrm{~m}$. Esses resultados corroboram com resultados encontrados por KAISER et al. (2009) que em um Latossolo, verificaram que a aeração do solo passou a 
Tabela 1 - Densidade do solo (Ds), densidade do solo relativa (Dsrel), macroporosidade, microporosidade e porosidade total em função dos estados de compactação em três profundidades.

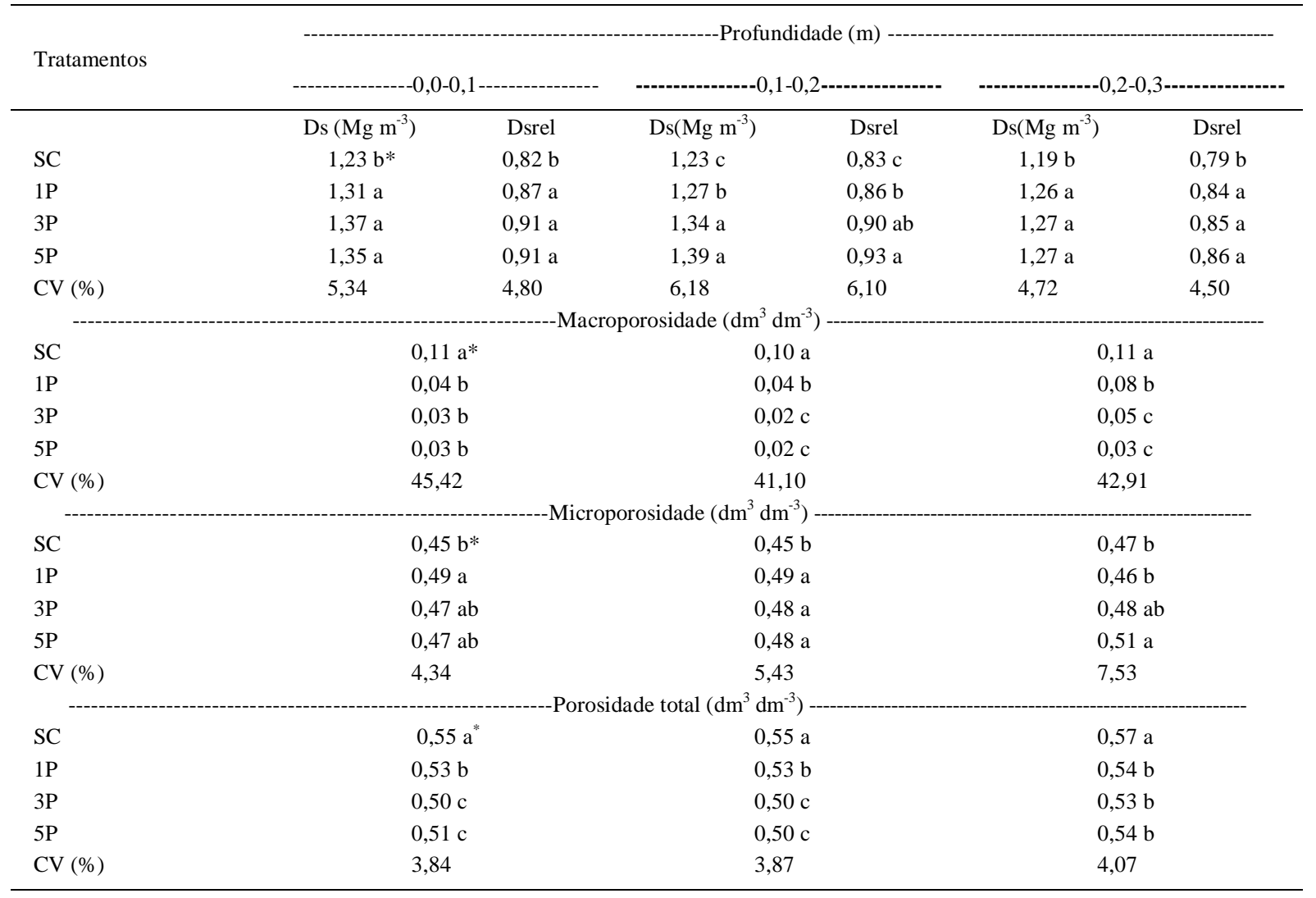

*Médias de tratamentos seguidas de mesma letra na coluna não diferem entre si pelo teste $\mathrm{t}$-Student $(\mathrm{P}<0,05)$. SC-Tratamento sem compactação com o rolo compactador, 1P-tratamento com uma passada do rolo, 3P-tratamento com três passadas do rolo e 5P-tratamento com cinco passadas do rolo. CV - coeficiente de variação.

ser limitante com valores de Ds acima de $1,32 \mathrm{Mg} \mathrm{m}^{-3}$. Nota-se que tanto o volume de Map quanto os valores de Ds foram semelhantes no 3P e 5P, demonstrando que as duas passadas a mais do $5 \mathrm{P}$ em relação ao $3 \mathrm{P}$ não contribuiram para um aumento significativo do estado de compactação.

O volume de Mip no SC foi inferior e diferiu $(\mathrm{P}<0,05)$ do 1P nas profundidades de $0,0-0,1$ e $0,1-0,2 \mathrm{~m}$ (Tabela 1). Para a profundidade de $0,1-0,2 \mathrm{~m}$, o volume de Mip no SC diferiu dos tratamentos 1P, 3P e 5P, os quais sofreram incrementos de $8,9,6,7$ e $6,7 \%$, respectivamente, já na profundidade de 0,2-0,3 ocorreu diferença estatística $(\mathrm{P}<0,05)$ quando se verifica os valores encontrados nos tratamentos $\mathrm{SC}$ e $1 \mathrm{P}$ em relação ao tratamento 5P (Tabela 1). Estes resultados concordam parcialmente com os resultados encontrados por BEUTLER \& CENTURION (2004), os quais também verificaram que a Mip não foi afetada significativamente pelo aumento da Ds.

Para a Pt observou-se redução nos tratamentos trafegados em relação ao não trafegado
(Tabela 1). O maior valor encontrado no tratamento SC diferiu estatisticamente $(\mathrm{P}<0,05)$ dos demais tratamentos em todas as profundidades. No entanto, nas profundidades de $0,0-0,1$ e $0,1-0,2 \mathrm{~m}$, observa-se que a $\mathrm{Pt}$ do 1Pé superior e difere do tratamento 5P, enquanto que na profundidade de $0,2-0,3 \mathrm{~m}$ os tratamentos $1 \mathrm{P}, 3 \mathrm{P}$ e $5 \mathrm{P}$ possuem valores semelhantes. COLLARES et al. (2008) trabalhando em um Latossolo argiloso, com tratamentos envolvendo sistema plantio direto com e sem passagem do rolo compactador e escarificado, verificou que a Pt não diferiu significativamente entre os tratamentos.

Com relação à taxa de infiltração de água no solo, o 5P diferiu significativamente dos tratamentos SC, 1P e 3P, com redução de $97 \%$ em relação ao tratamento SC, sendo que os tratamentos $1 \mathrm{P}$ e SC apresentaram taxa de infiltração de água no solo semelhantes, conforme pode ser observado na tabela 2. Esses valores corroboram aos encontrados por MILANI(2005), que variaram de $48 \mathrm{~mm} \mathrm{~h}^{-1}$ (valor mínimo) 
Tabela 2 - Taxa de infiltração de água no solo (Ti) e produtividade da cultura de trigo em quatro estados de compactação do solo.

\begin{tabular}{lll}
\hline Tratamentos & $\mathrm{Ti}\left(\mathrm{mm} \mathrm{h}^{-1}\right)$ & $\begin{array}{c}\text { Produtividade } \\
\left(\mathrm{Mg} \mathrm{ha}^{-1}\right)\end{array}$ \\
\hline $\mathrm{SC}$ & $377,00 \mathrm{a} *$ & $2.12 \mathrm{a} *$ \\
1P & $279,20 \mathrm{a}$ & $1.86 \mathrm{ab}$ \\
3P & $111,40 \mathrm{c}$ & $1.81 \mathrm{ab}$ \\
SP & $12,12 \mathrm{~d}$ & $1.64 \mathrm{~b}$ \\
$\mathrm{CV}(\%)$ & 83 & 7,83 \\
\hline
\end{tabular}

*Médias de tratamentos seguidas de mesma letra na coluna, não diferem entre si pelo teste t-Student $(\mathrm{P}<0,05)$. SC-Tratamento sem compactação com o rolo compactador, 1P-tratamento com uma passada do rolo, 3P-tratamento com três passadas do rolo e 5Ptratamento com cinco passadas do rolo. CV - coeficiente de variação.

a $396 \mathrm{~mm} \mathrm{~h}^{-1}$ (valor máximo), tendo em vista se tratar do mesmo Latossolo deste estudo.

O 1P apresentou uma elevada taxa de infiltração de água no solo. Uma possível explicação é a de que com apenas uma passada do rolo compactador não tenha proporcionado pressão suficiente para deformação dos bioporos, quando comparado aos tratamentos com maiores freqüência de tráfego (3P e 5P). SILVA et al. (2009), trabalhando em dois Latossolos sob diferentes estados de compactação, observaram que o estado de compactação não interferiu na taxa de infiltração inicial e estável, nem na lâmina de água infiltrada acumulada de cada solo devido à presença de bioporos.

Quanto à produtividade da cultura de trigo nos quatro estados de compactação do solo, os resultados demonstram que o tratamento $\mathrm{SC}$ proporcionou a maior produtividade entre os tratamentos, embora não diferindo dos tratamentos $1 \mathrm{P}$ e 3P, porém com diferença para o tratamento 5P, o qual apresentou produtividade $23 \%$ inferior ao tratamento SC. Isso demonstra que, após 8 anos de cultivo sob sistema plantio direto, os valores de Ds estão próximos ou dentro da faixa restritiva de produção, haja vista a não ocorrência de diferença entre os tratamentos SC, 1Pe 3P (Tabela 2).

Verifica-se que somente após cinco passadas com o rolo compactador houve efeito negativo na produtividade do trigo. A menor produtividade do tratamento $5 \mathrm{P}$, ocorreu em consequiência da modificação do estado estrutural do solo, principalmente o aumento da Ds e redução do volume de map, importantes atributos que definem resistência à penetração, infiltração de água e trocas gasosas no solo. SECCO et al. (2009), trabalhando em dois Latossolos argilosos também observaram decréscimo na produtividade de trigo quando a Ds foi de $1,50 \mathrm{Mg} \mathrm{m}^{-3}$. De acordo com REICHERT et al. (2009), esses valores de Ds encontrados seriam restritivos para Latossolos.

A figura 1 demonstra o modelo de regressão (polinomial de segundo grau) que mais se ajustou aos resultados da produtividade do trigo em função da Dsrel. A máxima produtividade do trigo para o intervalo avaliado foi obtida com valor de Dsrel de 0,83. Esse valor encontrado corrobora com valores propostos por LINDSTRON \& VOORHEES (1994) e é intermediário aos valores encontrados na literatura (BEUTLER et al., 2005; KLEIN, 2006), demonstrando que ainda faltam estudos complementares quando se relaciona estados de compactação e produtividade das culturas.

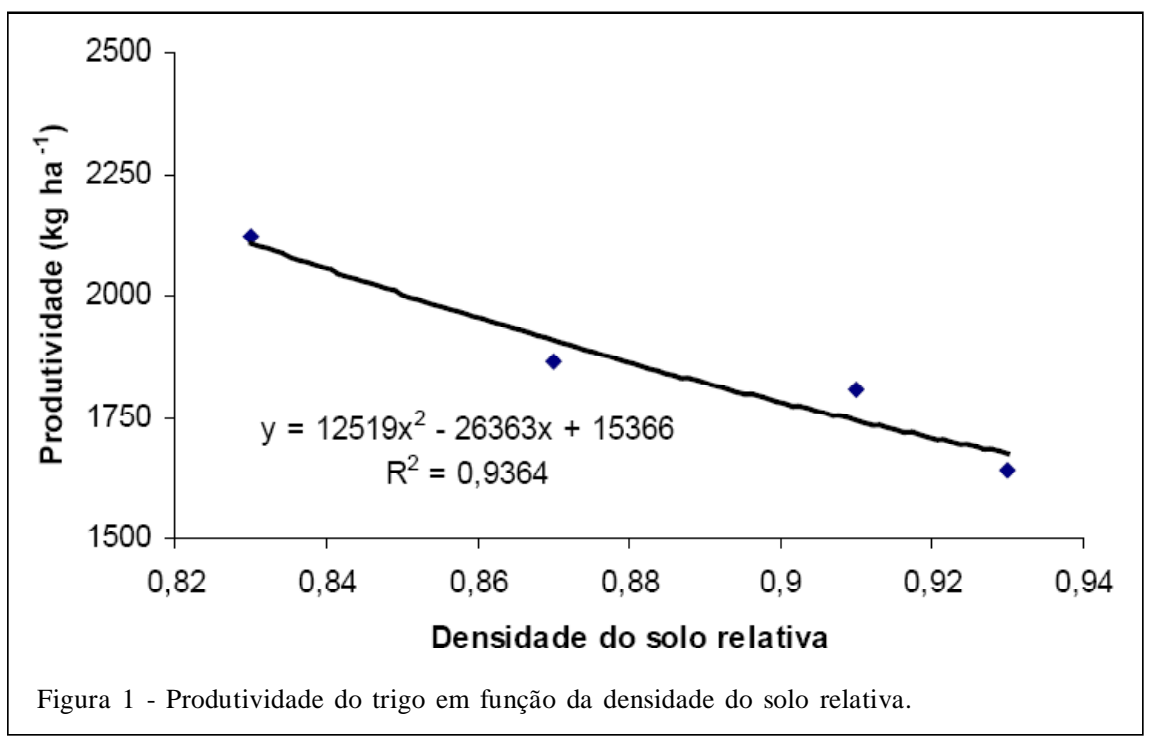

Ciência Rural, v.41, n.9, set, 2011. 


\section{CONCLUSÃO}

Os níveis de compactação proporcionados após três e cinco passadas com o rolo compactador não diferiram e afetaram negativamente a Ds e a Map. A compactação causada pelo 5P causou redução de $23 \%$ e $97 \%$, respectivamente, na produtividade de trigo e na taxa de infiltração de água no solo quando comparado ao SC. A Dsrel que proporcionou maior rendimento de grãos de trigo foi de 0,83 .

\section{REFERÊNCIAS}

ABNT-ASSOCIAÇÃO BRASILEIRA DE NORMAS TÉCNICAS. Solo: ensaio de compactação - Procedimento. NBR 7182/86. Rio de Janeiro, 1986. 10p.

BANZATTO, D.A.; KRONKA, S.N. Experimentação agrícola. 3.ed. Jaboticabal: FUNEP, 1995. 247p.

BERNARDO, S. Manual de irrigação - Infiltração de água no solo. Viçosa, MG: Universidade Federal de Viçosa, 1995. 657p.

BEUTLER, A.N.; CENTURION, J.F. Compactação do solo no desenvolvimento radicular e na produtividade da soja. Pesq Agropec Bras, v.39, p.581-588, 2004. Disponível em: <http:/ /www.scielo.br/pdf/pab/v39n6/v39n6a10.pdf >. Acesso em: 05 jul. 2011. doi: 10.1590/S0100-204X2004000600010.

BEUTLER, A.N. et al. Densidade relativa ótima de Latossolos vermelhos para a produtividade de soja. R Bras Ci Solo, v.29, p.843-849, 2005. Disponível em: <http://www.scielo.br/pdf/ rbcs/v29n6/28953.pdf>. Acesso em: 05 jul. 2011. doi: 10.1590/ S0100-06832005000600002.

COLLARES, G. L. et al. Compactação de um Latossolo induzida pelo tráfego de máquinas e sua relação com o crescimento e produtividade de feijão e trigo. R Bras Ci Solo, v.32, p.933942, 2008. Disponível em: <http://www.scielo.br/pdf/rbcs/ v32n3/a03v32n3.pdf>. Acesso em: 05 jul. 2011. doi:10.1590/ S0100-06832008000300003.

EMPRESA BRASILEIRA DE PESQUISA AGROPECUÁRIA EMBRAPA. Manual de métodos de análises de solo. 2.ed. Rio de Janeiro: Ministério da Agricultura e do Abastecimento, 1997. 212p.

EMPRESA BRASILEIRA DE PESQUISA AGROPECUÁRIAEMBRAPA. Centro Nacional de Pesquisa de Solos. Sistema brasileiro de classificação de solos. 2.ed. Brasília: Embrapa Produção da Informação, 2006. 306p.

KAISER, D.R. et al. Intervalo hídrico ótimo no perfil explorado pelas raízes de feijoeiro em um latossolo sob diferentes níveis de compactação. R Bras Ci Solo, v.33, p.845-855, 2009. Disponível em: 〈http://www.scielo.br/pdf/rbcs/v33n4/09.pdf >. Acesso em 05 jul. 2011. doi:10.1590/S010006832009000400009 .
KLEIN, V.A. Densidade relativa - um indicador da qualidade física de um Latossolo vermelho. R Ci Agroveterinárias, v.5, p.26-32, 2006. Disponível em: <http://rca.cav.udesc.br/ rca_2006_1/klein.pdf>. Acesso em: 05 jul. 2011.

LINDSTROM, M.J.; VOORHEES, W.B. Response of temperate crops in North America to soil compaction. In: SOANE, B.D.; OUWERKERK, C. van (Eds.). Soil compaction in crop production. London: Elsevier, 1994. Cap.12, p.265-286. (Developments in Agricultural Engineering, 2).

MILANI, D.I.C. Variabilidade espacial da infiltração de água e de atributos físicos em um Latossolo sob sistema plantio direto. 2005. 124f. Dissertação (Mestrado em Engenharia Agrícola) - Universidade Estadual do Oeste do Paraná, Cascavel, PR.

PIMENTEL GOMES, F.; GARCIA, C.H. Estatística aplicada a experimentos agronômicos e florestais: exposição com exemplos e orientações para o uso de aplicativos. Piracicaba: FEALQ, 2002. 309p.

REICHERT, J.M. et al. Reference bulk density and critical degree-of-compactness for no-till crop. production in subtropical highly weathered soils. Soil Till Res, v.102, p.242-254, 2009. Disponível em: <http://www.sciencedirect.com/science/article/ pii/S0167198708001025>. Acesso em: 05 jul. 2011. doi:10.1016/j.still.2008.07.002.

REUNIÃO DA COMISSÃO CENTRO-SUL BRASILEIRA DE PESQUISA DE TRIGO E TRITICALE. Informações técnicas da Comissão Centro-Sul Brasileira de Pesquisa de Trigo e Triticale para a safra de 2005. Londrina-PR: Embrapa Soja, 2005. 234p. (Embrapa Soja. Sistemas de produção, 7).

SECCO, D. et al. Atributos físicos e rendimento de grãos de trigo, soja e milho em dois Latossolos compactados e escarificados. Cienc Rural, v.39, p.58-64, 2009. Disponível em: <http:// w w w.s c i e 1 o.br/s c i e 1 o.ph p ? pid=S 0103 $84782009000100010 \&$ script $=$ sci_abstract\&tlng=pt $>$. Acesso em: 05 jul. 2011. doi:10.1590/S0103-84782009000100010.

SILVA, V.R. Propriedades físicas e hídricas em solos sob diferentes estados de compactação. Santa Maria. 2003. 171f. Tese (Doutorado em Agronomia) - Universidade Federal de Santa Maria, RS.

SILVA, V.R. da et al. Soil water dynamics related to the degree of compatation of two brasilian oxisols under no-till sistem. R Bras Ci Solo, v.33, p.1097-1104, 2009. Disponível em: <http://www.scielo.br/scielo.php?pid=S0100$06832009000500003 \&$ script $=$ sci_arttext $>$. Acesso em: 05 jul. 2011. doi:10.1590/S0100-06832009000500003.

SUZUKI, L.A.S. et al. Grau de compactação, propriedades físicas e rendimento de culturas em Latossolo e Argissolo. Pesq Agropec Bras, v.42, p.1159-1167, 2007. Disponível em: <http://www.scielo.br/pdf/pab/v42n8/a13v42n8.pdf>. Acesso em: 05 jul. 2011. doi:10.1590/S0100-204X2007000800013. 